\title{
Nocturnal blood pressure patterns and cardiac damage: there is still much to learn
}

\author{
Domenico Di Raimondo ${ }^{1}$. Gaia Musiari ${ }^{1}$ - Antonio Pinto ${ }^{1}$
}

Received: 1 November 2019 / Revised: 5 November 2019 / Accepted: 6 November 2019 / Published online: 12 December 2019

(c) The Japanese Society of Hypertension 2019

Most normotensive and hypertensive subjects show a physiologic reduction in blood pressure (BP) during sleep. However, a minority of subjects show a blunted or exaggerated nocturnal BP reduction or even a paradoxical increase in BP. Over the past 30 years, 24-h ambulatory blood pressure monitoring (ABPM) has been suggested to be the recommended technique for out-of-office BP measurements, helping to significantly improve the clinical management of hypertension, precisely because it may provide several pieces of information regarding nocturnal BP levels otherwise not available [1].

Ascertaining whether the "dipping" status (i.e., the nocturnal decline in BP) is maintained or the circadian rhythm of $\mathrm{BP}$ is altered (falling into one of the three alternative nocturnal BP patterns described as nondipping, extreme dipping and reverse dipping) (see Fig. 1) is highly relevant since both the dipping status and the related night/day BP ratio are recognized as better predictors of all-cause mortality and adverse cardiovascular (CV) outcomes than inoffice BP or daytime ambulatory BP [1].

An impressive amount of evidence based on several well-designed prospective studies has confirm that a reduced nocturnal BP decline has been associated with a high risk of death, with a poor $\mathrm{CV}$ outcome and highly advanced target organ damage [2].

Trying to classify hypertensive individuals into subgroups based on their nocturnal BP patterns is not free of criticism, the most relevant of which is the poor reproducibility of the dipping status [2]. Furthermore, there are substantial methodological issues due to the dichotomic classification of dipping/nondipping often used in papers,

Domenico Di Raimondo

domenico.diraimondo@unipa.it

1 Division of Internal Medicine and Stroke Care, Department of Promoting Health, Maternal-Infant. Excellence and Internal and Specialized Medicine (Promise) G. D'Alessandro, University of Palermo, Piazza delle Cliniche 2, 90127 Palermo, Italy which appears to be much less specific in predicting $\mathrm{CV}$ outcomes than the classification with four patterns [3].

When considering four dipping subgroups, the responsibility for a very substantial percentage of those with the nondipping pattern who have the worst prognoses (for all outcomes) is attributable to reverse dippers [2]. On the other hand, extreme dippers seem to have little or no risk, showing a good prognostic value that is as good as those with a dipping pattern, if not better, [3-5] making the actual prognostic value of extreme dipping worthy of targeted analyses. Note that a consistent part of the cardio and cerebrovascular risk ascribed to the extreme dipping pattern may be related to nocturnal recurrent episodes of low BP exacerbated by an inappropriate dose of antihypertensive therapy at bedtime. This risk further underlines the importance of establishing the nocturnal BP pattern before setting a drug therapy regimen, especially in the highly fragile categories of patients (those with elderly, secondary prevention of chronic coronary syndromes, acute and chronic cerebrovascular diseases) $[3,5]$.

The assessment of hypertensive heart disease was often limited to an analysis of the direct consequences of the chronically increased afterload on left ventricular (LV) chambers, leading to LV hypertrophy, left atrial enlargement, and LV systolic and diastolic dysfunction, all of which are risk factors for the development of heart failure and atrial arrhythmias. Recently, a study of the complex and various influences on cardiac structure and function that occur secondary to prolonged untreated systemic hypertension has led to the observation that the right heart is often affected, although the right chambers are not directly exposed to the chronic increase in the systemic afterload, and the architecture and the pattern of contractions is intrinsically different between the right and left ventricles. The involvement of the right ventricle (RV) and then atrium is pathophysiologically justified by mechanic causes (mainly through the interventricular septum remodeling that leads to a direct left-to-right transmission of the systemic afterload) and by the neurohumoral activation induced by 
Fig. 1 Main characteristics of the four nocturnal blood pressure patterns.

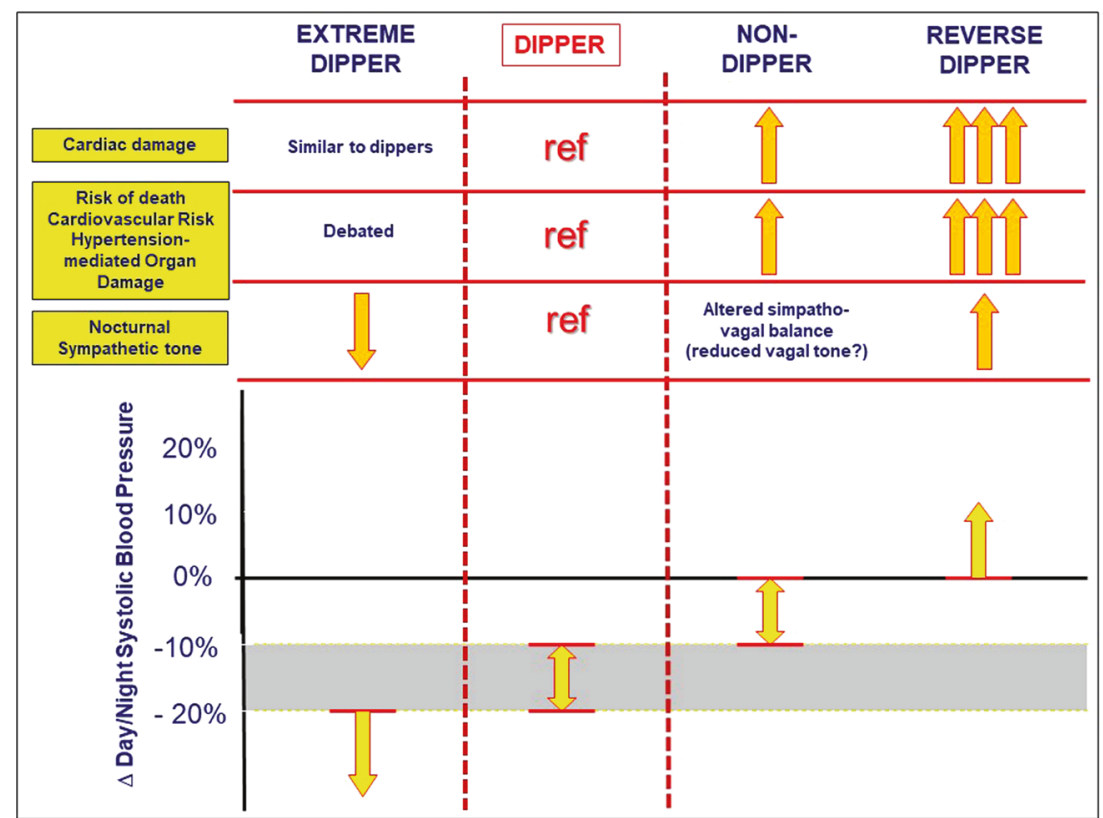

arterial hypertension that can influence mainly the left, but also the right, ventricle structure and function. For these reasons, right ventricular hypertrophy may be found in approximately one-fifth of the untreated patients with essential hypertension in a manner that is not dependent on the development of pulmonary hypertension [6]. The extent of the hypertension-mediated organ damage (HMOD) seems to be related to the nocturnal dipping of BP as well [7] as the LV and RV structures and mechanics; likewise, the diastolic and global functions have been reported to be adversely affected by a blunted nocturnal BP reduction [8], although only a few authors tried to ascertain the impact of nocturnal BP patterns on RV function and mechanics.

To further improve our understanding of the relationship between RV mechanics and nocturnal BP, Tadic et al. [9] evaluated 190 untreated hypertensive patients with matching two-dimensional echocardiographic data and included an analysis of right ventricular global and free wall longitudinal strains to the nocturnal BP patterns obtained through the ABPM data. Among the four groups (extreme dippers, dippers, nondippers, and reverse dippers) with similar 24-h and daytime BP values, the structure and the systolic and diastolic functions of the RV did not significantly differ; interestingly, RV remodeling progressively deteriorated from the extreme dippers to the reverse dippers. After the multivariate logistic regression analysis that considered any possible confounding factors, such as age, sex, 24-h systolic BP, LV mass index, RV wall thickness, and E/ $\mathrm{e}^{\prime}$ ratio, only the reverse dipping pattern of BP was independently associated with RV remodeling. These results substantially agree the previous reports on LV remodeling: the extent of cardiac damage gradually increases from the extreme dippers to the dippers, the nondippers, and the reverse dippers, but a statistically significant difference is reached only when comparing the reverse dippers to the rest of the hypertensive patients [8], underlying the strong need to assess (and eventually correct) the reverse dipping pattern.

In conclusion, the findings provided by Tadic et al. support the latest recommendations to record BP over a whole 24-h period to ensure a better stratification of HMOD in clinical practice, a closer follow-up in select high-risk populations (nondipper/reverse dipper), and more appropriate action for restoring a normal dipping pattern (bedtime antihypertensive therapy administration), when appropriate. Future studies are needed to examine how the impaired circadian rhythm of BP and which of the main determinants of nocturnal BP (abnormal sympatho-vagal balance [10], excessive fluid retention, and others) may influence the development of four-chamber cardiac damage as well as the ability of antihypertensive treatment to restore right chamber morphology and function. Finally, there is a substantial lack of evidence regarding improvements in the prognosis of those with reverse dipping patterns after the restoration of physiological nocturnal dips of BP. Researchers are strongly called on to answer these questions.

\section{Compliance with ethical standards}

Conflict of interest The authors declare that they have no conflict of interest. 
Publisher's note Springer Nature remains neutral with regard to jurisdictional claims in published maps and institutional affiliations.

\section{References}

1. Williams B, Mancia G, Spiering W, Agabiti Rosei E, Azizi M, Burnier M, et al. Authors/Task Force Members. 2018 ESC/ESH Guidelines for the management of arterial hypertension: The Task Force for the management of arterial hypertension of the European Society of Cardiology and the European Society of Hypertension: the Task Force for the management of arterial hypertension of the European Society of Cardiology and the European Society of Hypertension. J Hypertens. 2018;36:1953-2041.

2. Yang WY, Melgarejo JD, Thijs L, Zhang ZY, Boggia J, Wei FF, et al. International Database on Ambulatory Blood Pressure in Relation to Cardiovascular Outcomes (IDACO) Investigators. Association of office and ambulatory blood pressure with mortality and cardiovascular outcomes. JAMA. 2019;322:409-20.

3. Fagard RH, Thijs L, Staessen JA, Clement DL, De Buyzere ML, De Bacquer DA. Night-day blood pressure ratio and dipping pattern as predictors of death and cardiovascular events in hypertension. J Hum Hypertens. 2009;23:645-53.

4. Salles GF, Reboldi G, Fagard RH, Cardoso CR, Pierdomenico $\mathrm{SD}$, Verdecchia P, et al. Prognostic effect of the nocturnal blood pressure fall in hypertensive patients: the ambulatory blood pressure collaboration in patients with hypertension $(\mathrm{ABC}-\mathrm{H})$ meta-analysis. Hypertension. 2016;67:693-700.
5. Di Raimondo D, Miceli G, Casuccio A, Tuttolomondo A, Buttà C, Zappulla V, et al. Does sympathetic overactivation feature all hypertensives? Differences of sympathovagal balance according to night/day blood pressure ratio in patients with essential hypertension. Hypertens Res. 2016;39:440-8.

6. Cuspidi C, Sala C, Muiesan ML, De Luca N, Schillaci G, Working Group on Heart, Hypertension of the Italian Society of Hypertension. Right ventricular hypertrophy in systemic hypertension: an updated review of clinical studies. J Hypertens. 2013;31:858-65.

7. Gong S, Liu K, Ye R, Li J, Yang C, Chen X. Nocturnal dipping status and the association of morning blood pressure surge with subclinical target organ damage in untreated hypertensives. J Clin Hypertens. 2019;21:1286-94.

8. Ivanovic BA, Tadic MV, Celic VP. To dip or not to dip? The unique relationship between different blood pressure patterns and cardiac function and structure. J Hum Hypertens. 2013;27:62-70.

9. Tadic M, Cuspidi C, Sljivic A, Pencic B, Mancia G, Bombelli M, et al. Do reverse dippers have the highest risk of right ventricular remodeling? Hypertens Res. 2019. https://doi.org/10.1038/ s41440-019-0351-2. [Epub ahead of print].

10. Di Raimondo D, Musiari G, Grova M, Miceli G, Tuttolomondo A, Pinto A. The "neurocentric" approach to essential hypertension: how reliable is the paradigm of hyperkinetic hypertension? A focus on the sympathetic nervous system dysregulation in essential hypertensive patients with elevated resting heart rate. Curr Pharm Des. 2017;23:4635-49. 